\title{
THE MIRAS “ALL-LICEF” CALIBRATION MODE
}

\author{
Ignasi Corbella ${ }^{(1)}$, Verónica González-Gambau ${ }^{(2)}$, Francesc Torres ${ }^{(1)}$, Nuria Duffo $^{(1)}$, \\ Israel Durán $^{(1)}$, Manuel Martín-Neira ${ }^{(3)}$
}

(1) Remote Sensing Laboratory, Universitat Politècnica de Catalunya (UPC).

C/ Jordi Girona 1-3. 08034 Barcelona, Spain.

(2) Department of Physical Oceanography, Institute of Marine Sciences, CSIC

and Barcelona Expert Center, Passeig Maritim de la Barceloneta, 37-49, 08003 Barcelona, Spain.

(3) European Space Agency (ESA-ESTEC) Noordwijk. The Nederlands

contact e-mail: corbella@tsc.upc.edu

\section{INTRODUCTION}

After more than six years of operation, the SMOS (Soil Moisture and Ocean Salinity) European Space Agency (ESA) mission [1] has proven to be highly useful for a variety of scientific applications related to soil moisture over land, ocean salinity and winds over ocean and specific studies over ice covered surfaces. Level 2 data is currently used by many institutions around the world to extract relevant information aimed at improving weather forecast, extreme events monitoring and others.

SMOS single payload is the Microwave Imaging Radiometer using Aperture Syntyhesis (MIRAS), an interferometric radiometer consisting of an array of 69 low-gain dual-polarization antennas placed along the three arms of a Y-shaped structure [2]. The fundamental measurement of the instrument is the visibility function [3] in units of Kelvin, obtained after amplitude and phase calibration of the complex cross-correlation between the signals collected by all pairs of antennas. A given sample of this function depends on the relative spatial coordinates of one antenna with respect to the other in a pair. The particular case of both antennas collapsing in a single location is the zero-spacing visibility, which is equal to the antenna noise temperature for this particular element. The MIRAS instrument is designed to use three redundant Noise Injection Radiometers (NIR) specifically to measure this average antenna temperature. The SMOS Level 1 operational processor (L1OP) uses these three measurements in the image reconstruction part. Nevertheless, in its latest version only one of the three NIRs is used, as it shows improved seasonal and long-term stability with respect to using all three. Additionally, the NIRs are used in the calibration of the power measurement system (PMS) installed in all

Work supported in part by the European Space Agency with Deimos Enginheria (Portugal) under SMOS P7 subcontract DME CP12 no. 2015005 and $\mathrm{CCN}-1$; and by the Spanish Ministry of Science and Technology (MCYT) projects TEC2014- 58582-R. receivers [4].

This paper describes a different scheme in which SMOS data is entirely processed without using the NIR outputs. In principle it is intended as a backup solution in case of failure or malfunction of all three NIR and it will be included in the next version of the L1OP (v700). It is, however, already implemented in the MIRAS testing software (MTS) [5] and has been called "all-LICEF mode" in reference to the name given to the MIRAS individual receivers: "LIghtWeight CostEffective Front-end".

\section{THE ALL-LICEF MODE}

All three NIRs are located in the center of the Y-shaped array and are used for two different purposes in the data processing chain:

- Measuring the power of the noise sources, needed in the internal calibration to retrieve the individual power gain of the receivers' PMS.

- Measuring the scene antenna temperature, which in turn is used for getting the visibility at zero spacing

These two usages are analyzed below.

\subsection{Power gain calibration}

The first usage can be covered if the receivers' gain is directly calibrated using the cold sky during external calibration. The possibility of turning the spacecraft in order to look towards the cold sky was only considered in the SMOS project in a quite late stage. Gain calibration was designed to be carried out only from internal noise injection [6], hence the need of measuring, using the NIRs, the actual noise provided by the sources. However, as long as the possibility of external calibration became feasible, a new PMS calibration approach based only on cold sky measurements was suggested 
[7]. At that time it was considered only as a backup or validation option and, although the corresponding sequence was indeed programmed in the payload onboard software and it is presently activated in a regular basis, its processing was not implemented in the SMOS operational L1 processor. Figure 1 shows a block diagram of the PMS gain calibration strategy, both for the nominal processing (internal calibration plus NIR) and for the proposed all-LICEF mode. The nominal internal PMS calibration uses the NIR measurement as a secondary calibration standard, which is in turn calibrated using the sky, including the values of front-end losses $\left(L_{1}\right)$ adjusted to correct for drifts [8]. To compensate the distribution network unbalance, a correction using the so-called "CAS factors" was introduced in the processing [9]. A new set of these factors must be computed only once whenever there is a change in MIRAS operation (for example switching components from nominal to redundant). In measurement mode, the plane translation from internal calibration plane (CIP) to antenna plane (HAP/VAP) uses again the front-end losses obtained from external calibration. In the case of all-LICEF, the PMS gain at HAP/VAP is directly recovered from the values obtained in the external calibration. Overall, the process is much simpler.

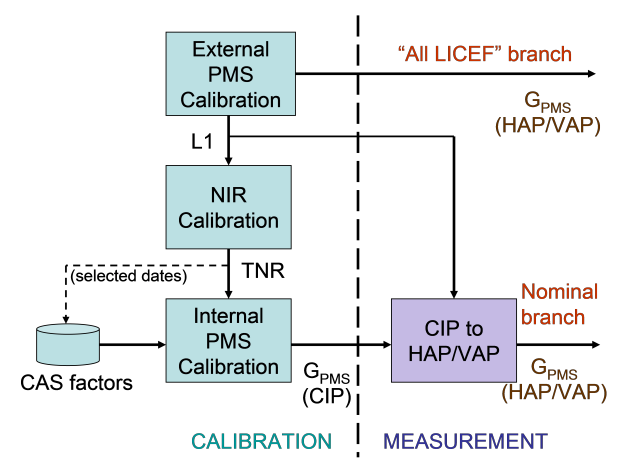

Fig. 1. Block diagram of the nominal and "all LICEF" PMS calibration strategies

The MIRAS Testing Software [5] fully processes the PMS external calibration sequences and regularly provides PMS gains both from external and internal calibration sequences. Figure 2 shows the average PMS gain along all receivers as a function of time during the entire mission up to the present. To make the comparison consistent, all gains are referenced to the internal calibration plane (CIP). The "all-LICEF" mode predicts a lower long-term drift, probably due to being insensitive to drifts of the noise injection network. The CAS correction factors were updated at November 2009, January 2010 and January 2011, corresponding to switching some command and monitoring nodes from nominal to redundant operation due to failure.

\subsection{Antenna temperature measurement}

Concerning the main use of the NIR -measurement of the scene antenna temperature- it can be carried out directly by each individual LICEF operating as a total power radiometer. This implies a complete calibration of the PMS including both gain and receiver noise temperature. As explained in the previous section, it is obtained during the cold sky external calibration using standard radiometer calibration methods. In this approach a different and independent measurement of antenna temperature is obtained for each single receiver at each snapshot. Although each of them may have uncertainties due to thermal noise and gain fluctuations, their average turns out to be a neat estimation of the scene antenna temperature, with even less random fluctuations than the NIR measurements. Figure 3 shows the antenna temperatures measured by the NIR and by the LICEF-PMS for the ascending orbit over the pacific ocean depicted in the same figure. In both cases, the average along all the measurements (3 NIRs and 66 LICEFs respectively) are drawn. Extreme values for the case of the LICEF is also shown as dashed lines. As it is apparent from the figure, both measurements are highly mutually consistent, with some less noise in the case of the LICEF due to averaging. Both cases are affected by radio frequency interferences at high north latitudes.

\section{LONG- AND SHORT- TERM DRIFTS}

Detailed analyses of SMOS brightness temperature, especially over the Pacific Ocean, have shown that there exist non-negligible seasonal variations and differences between ascending and descending passes. To further assess the allLICEF operation, these differences have been computed for both modes: the nominal and the all-LICEF. In agreement with the SMOS ocean team, a set of descending and ascending orbits over a zone of the Pacific ocean with expected low seasonal variation was selected. Their temporal coverage goes from June 2010 to December 2012. For the present analysis only the data corresponding to latitudes ranging from -55 to 5 is considered. The stability assessment is carried out using as parameter the bias of $(\mathrm{Tx}+\mathrm{Ty}) / 2$ with respect to an ocean model provided by the SMOS ocean salinity science team. The use of the first Stokes parameter avoids uncertainties due to Faraday rotation. Temporal plots of reconstructed brightness temperature in the alias-free Field of View are produced with different lines for ascending and descending passes and shown in figure 4 for the nominal processing (using only one NIR antenna temperature) and for the all-LICEF mode. As it is apparent from the plots, the consistency between ascending and descending values and the seasonal stability is at least similar in both cases, even though a noise injection radiometer is in principle more stable than a total power radiometer. 


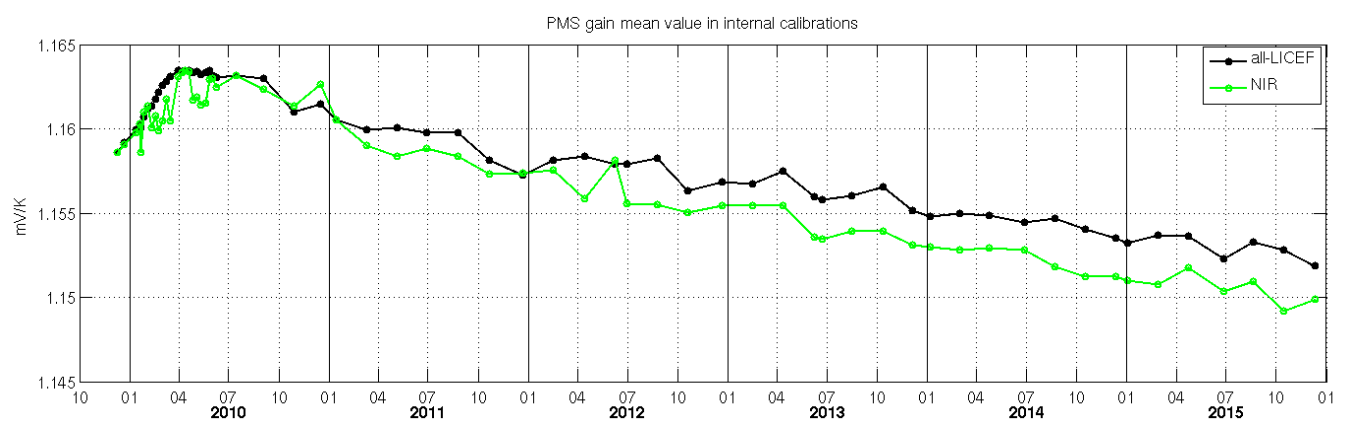

Fig. 2. Internal PMS gain computed using "all-LICEF" and NIR modes
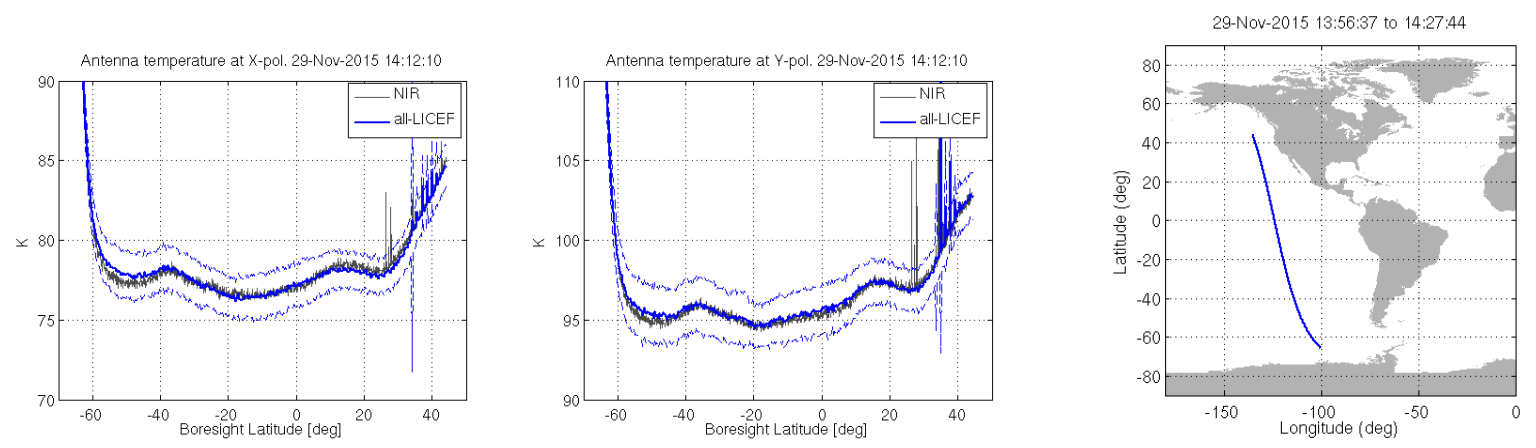

Fig. 3. Measured average antenna temperature of the orbit shown at right using "all-LICEF" (left) and NIR (center) modes.

\section{CONCLUSIONS}

Since each of the individual elements of the MIRAS array is a total power radiometer, the zero-spacing visibility can be obtained by the average of all the corresponding antenna temperatures. The main advantage of this option with respect to using the NIR measurements is that amplitude calibration is more consistent between zero-spacing visibility and the rest. On the other hand, total power radiometers are not usually as stable as noise injection radiometers, so a small loose of stability could be expected. Preliminary results show, however, similar performance.

In the nominal MIRAS operation, PMS gain is calibrated using noise injection through the a noise distribution network, requiring NIR to measure the injected noise power. In measurement mode the calibrated PMS gain is translated to antenna plane making the process very complex and prone to uncertainties due to various parameters measured on ground and used on flight with the assumption that they are constant. On the other hand, a direct PMS gain calibration at antenna plane provides accurate PMS gains ready for their use in measurement mode without needing the NIR.

The all-LICEF mode, which combines the external PMS gain calibration with the measurement of antenna temperature from the LICEFs PMS voltages, provides good results, in any case compatible with the nominal processing algorithms implemented in the L1OP.

\section{REFERENCES}

[1] Hubert Barré, Berthyl Duesmann, and Yann Kerr, "SMOS: The mission and the system," IEEE Transactions on Geoscience and Remote Sensing, vol. 46, no. 3, pp. 587-593, March 2008.

[2] Kevin McMullan, Michael Brown, Manuel Martín-Neira, W Rits, S Ekholm, J Marti, and Jerzky Lemanzyk, "SMOS: The payload," IEEE Transactions on Geoscience and Remote Sensing, vol. 46, no. 3, pp. 594-605, March 2008.

[3] Ignasi Corbella, Nuria Duffo, Mercè Vall-llossera, Adriano Camps, and Francesc Torres, "The visibility function in interferometric aperture synthesis radiometry," IEEE Transactions on Geoscience and Remote Sensing, vol. 42, no. 8, pp. 1677-1682, August 2004.

[4] Ignasi Corbella, Francesc Torres, Adriano Camps, Andreas Colliander, Manuel Martín-Neira, Serni Ribó, Kimmo Rautiainen, Nuria Duffo, and Mercè Vallllossera, "MIRAS end-to-end calibration. Application to SMOS L1 processor," IEEE Transactions on Geoscience and Remote Sensing, vol. 43, no. 5, pp. 1126-1134, May 2005.

[5] Ignasi Corbella, Francesc Torres, Nuria Duffo, Verónica González, Adriano Camps, and Mercè Vall-llossera, 

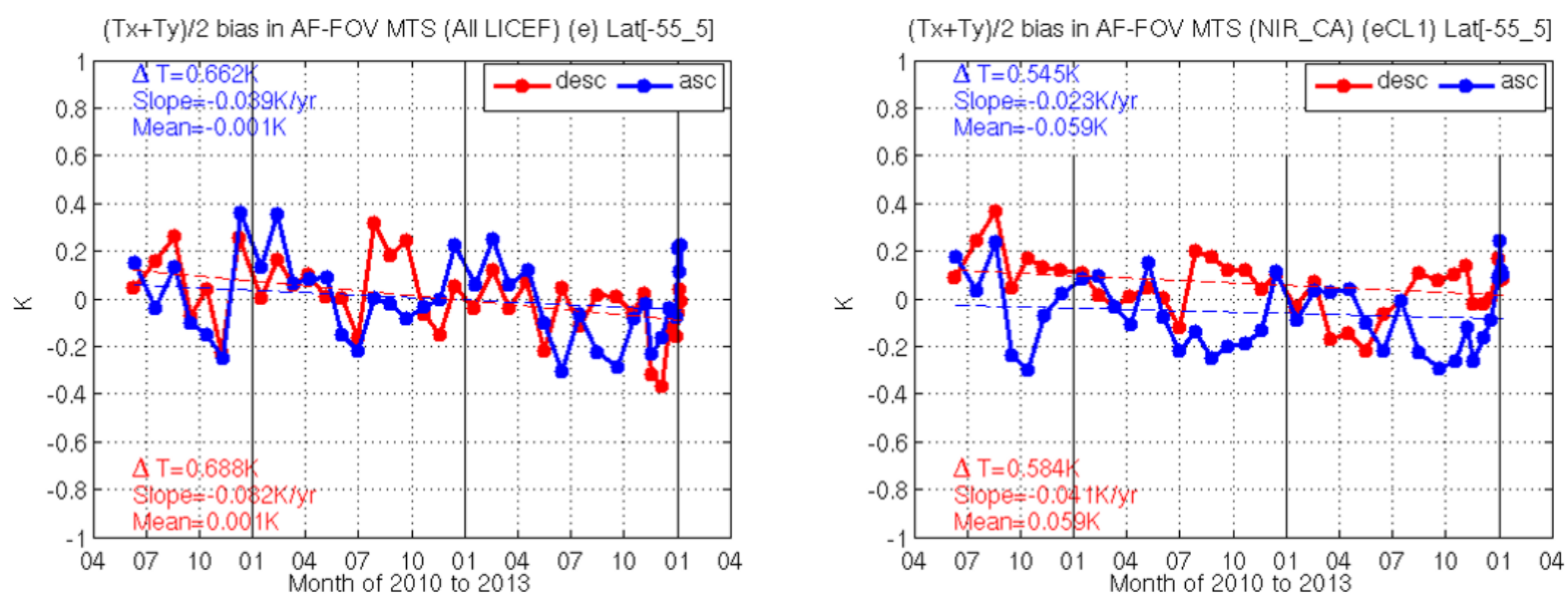

Fig. 4. Brightness temperature stability for "all-LICEF" (left) and NIR (right) modes.

"Fast processing tool for SMOS data," in International Geoscience and Remote Sensing Symposium, IGARSS 2008, Boston(Ma), USA, 7 - 11 July 2008, number II, pp. 1152-1155, IEEE.

[6] Francesc Torres, Adriano Camps, Javier Bará, Ignasi Corbella, and Roberto Ferrero, "On-board phase and modulus calibration of large aperture synthesis radiometers: Study applied to MIRAS," IEEE Transactions on Geoscience and Remote Sensing, vol. GRS-34, no. 4, pp. 1000-1009, July 1996.

[7] Francesc Torres, Verónica González-Gambau, and Cristina González-Haro, "One-point calibration in interferometric radiometers devoted to earth observation," in Proceedings of SPIE Europe Remote Sensing 2008, Cardiff. Wales, United Kingdom, 15-18 september 2008, SPIE Europe Remote Sensing 2008. Sensors, Systems, and Next-Generation Satellites XII.

[8] Ignasi Corbella, Francesc Torres, Nuria Duffo, Israel Durán, Miriam Pablos, and Manuel Martin-Neira, "Enhanced SMOS amplitude calibration using external target," in IEEE International Geoscience and Remote Sensing Symposium, IGARSS 2012, Munich, Germany, 22-27 July 2012, pp. 2868 - 2871, IEEE.

[9] Ignasi Corbella, Francesc Torres, Nuria Duffo, Verónica González-Gambau, Miriam Pablos, Israel Duran, and Manuel Martín-Neira, "MIRAS calibration and performance. results from the SMOS in-orbit commissioning phase," IEEE Transactions on Geoscience and Remote Sensing, vol. 49, no. 9, pp. 3147 -3155, September 2011. 